\section{Aplastic anaemia presenting with features of raised intracranial pressure}

\section{Aditi Mohla Olugbemisola Oworu Colin Hutchinson}

J R Soc Med 2006;99:315-316

Anaemia is a commonly encountered medical condition although retinopathy due to aplastic anaemia is not commonly seen.

\section{CASE HISTORY}

A 23-year-old male presented with a 1-week history of bilateral blurred vision, worse in the left eye. It was associated with frontal and temporal headache made worse by coughing. There was no preceding trauma or blood loss, he was on no medication and was not exposed to noxious chemicals or irradiation. He was not diabetic nor hypertensive. Prior to developing blurred vision, he had coryzal-like symptoms for 2 days but no myalgia, rash or joint pains. His maternal uncle died of a blood disorder and his maternal cousin has acute lymphoid leukaemia.

On examination, he was non obese but looked pale. He was haemodynamically stable with a heart rate of 82 beats/ min and a blood pressure of $150 / 56 \mathrm{mmHg}$. Visual acuity was 6/18 (right eye) and 6/60 (left eye). Anterior segments of both eyes were normal with no pupillary defect. Fundoscopy showed bilateral grossly swollen optic discs with scattered retinal haemorrhages more marked in the posterior pole and gross macular oedema in the left eye (Figure 1a,b).

A computerized tomography scan and magnetic resonance imaging of head and orbit showed no evidence of raised intracranial pressure, i.e. no space occupying lesion and normal ventricles. There were no meningeal infiltrations and optic nerves were normal. There were no features to suggest cerebral venous thrombosis.

Blood tests, including serum glucose, angiotensin converting enzyme, antineutrophilic cytoplasmic antibodies, syphilitic serology, anti-mitochondrial antibody, electrolytes and urea, folate and vitamin $\mathrm{B}_{12}$ were normal.

Full blood count revealed severe pancytopenia with haemoglobin of $5.8 \mathrm{~g} / \mathrm{dL}$, white cell count of $2.7 \times 10^{9} / \mathrm{L}$, platelet count of $15 \times 10^{9} / \mathrm{L}$ and mean corpuscular volume

Eye Department, Huddersfield Royal Infirmary, Lindley,

Huddersfield HD3 3EA, UK

Correspondence to: Olugbemisola Oworu

E-mail: gbemsol@hotmail.com (a)

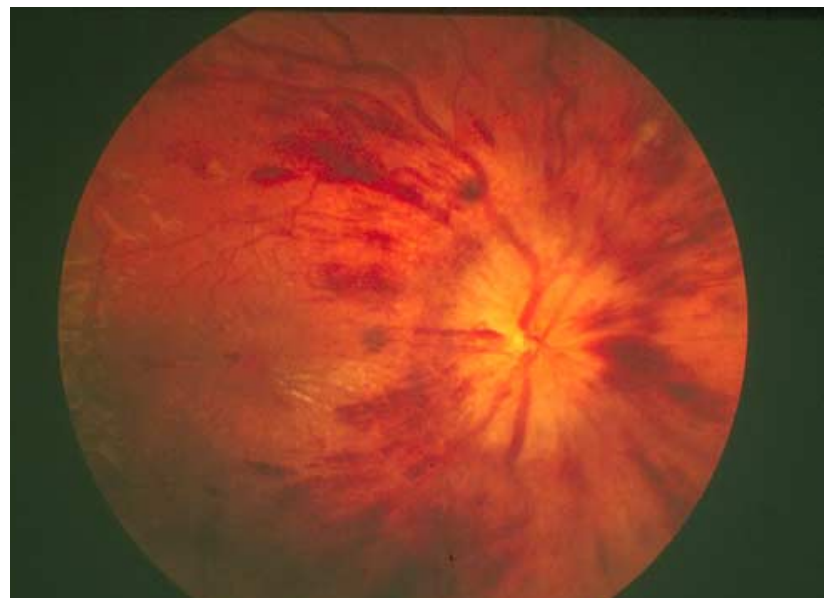

(b)

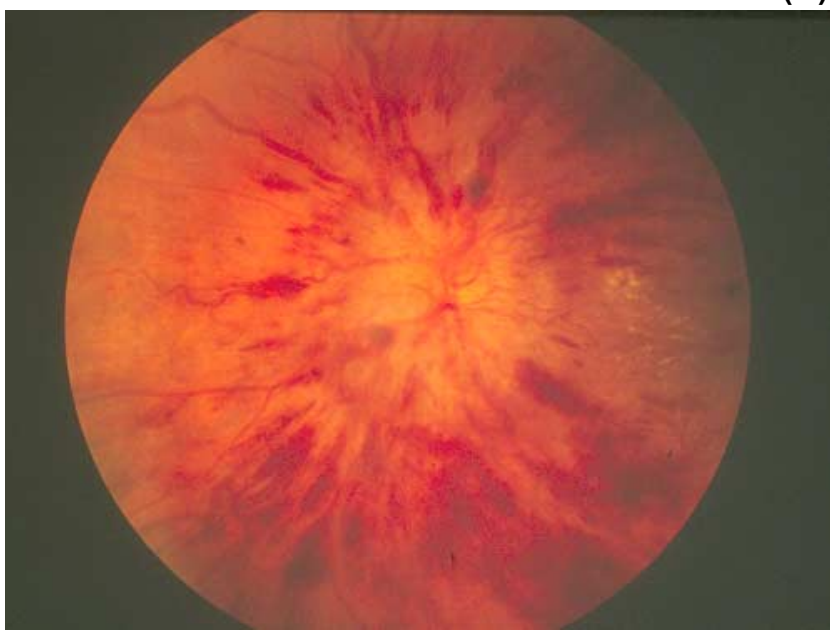

Figure 1 Findus picture at presentation: there are multiple superficial flame-shaped haemorrhages and areas of deep blotch haemorrhages in both (a) right eye and (b) left eye. (This figure can be seen in colour online)

of $109.8 \mathrm{fl}$. Blood film showed aplastic anaemia confirmed by a bone marrow biopsy.

He had packed cells and platelet transfusions initially and this was repeated due to insufficient endogenous haemopoiesis. He was subsequently commenced on antilymphocyte globulin and cyclosporin. As his haemoglobin improved, so did the retinopathy and visual acuity.

Ten months later, his haemoglobin stabilized at $15 \mathrm{~g} / \mathrm{dL}$ with immunosuppressive treatment alone. The retinopathy had almost completely resolved with only a residual deep blotch haemorrhage at the left macula. His visual acuity was $6 / 5$ right eye and 6/6 left eye.

\section{COMMENT}

Retinopathy associated with gastrointestinal haemorrhage and resolving following correction of the anaemia, was first reported by Ulrich in $1883 .{ }^{1}$ The manifestations of anaemic retinopathy are non-specific. Ophthalmic features include 
retinal haemorrhages, exudates, disc pallor, optic atrophy, neuritis, retinal detachment and papilloedema. ${ }^{1}$

The features of anaemic retinopathy seem to be universal, irrespective of the cause of the anaemia. The retinopathy also seems to resolve once the anaemia is corrected, as shown in the patient presented. It is widely believed that anaemia causes diminished capillary oxygenation, which increases the vessel wall permeability resulting in extravasation of blood products. ${ }^{2}$ There seems to be a direct correlation between the degree of anaemia and the severity of the retinopathy.

Aplastic anaemia is a life threatening condition. It usually presents with anaemia, bleeding and infection. It can be inherited but it is more commonly acquired. The ocular findings include cotton wool spots, nerve fibre layer or preretinal haemorrhages, vitreous haemorrhages and optic disc oedema.

Mansour et al. reported ocular findings in 18 patients with aplastic anaemia. ${ }^{3}$ In their report, the patients were known to be anaemic prior to the development of the retinopathy.

In our case, the patient certainly had disc oedema and nerve fibre layer haemorrhages with gross macular oedema, all of which resolved once the anaemia was corrected.

Some patients with aplastic anaemia also develop pseudotumour cerebi which may require treatment geared towards lowering the raised intracranial pressure. ${ }^{4-6}$ It has been suggested that papilloedema associated with aplastic anaemia could be due to increased intracranial pressure from anaemia-induced cerebral hypoxia. ${ }^{7}$

The presentation in this patient initially was suggestive of raised intracranial pressure. We feel it is important that clinicians are made aware of this unusual presentation and that this case might hopefully help direct treatment to the underlying cause.

\section{REFERENCES}

1 Weiss L. Anaemic retinopathy. Pennsylvania Med 1966;69:35-6

2 Koh A. Anaemia - more than meets the eye. Singapore Med J 1998;39: 222

3 Mansour AM, Salti HI, Han DP et al. Ocular findings in aplastic anaemia. Ophthalmologic 2000;214:399-402

4 Biousse V, Rucker JC, Vignal C, Crassard I, Katz BJ, Newman NJ. Anaemia and papilledema. Am J Ophthalmol 2003;135:437-46

5 Nazir SA, Siatkowski RM. Pseudotumor cerebri in idiopathic aplastic anemia. J Am Assoc Pediatr Ophthalmol Strabismus 2003;7:71-4

6 Jeng MR, Rieman M, Bhakta M, Helton K, Wang WC. Pseudotumor cerebri in two adolescents with acquired aplastic anemia. J Pediatr Hematol Oncol 2002;24:765-8

7 Lubeck MJ. Papilloedema caused by iron deficiency anaemia. Trans Am Acad Ophthalmol 1959;63:306
Severe ischaemic cardiomyopathy associated with khat chewing

\author{
Sagar Saha Clare Dollery
}

J R Soc Med 2006;99:316-318

Legal chewing of khat leaves is prevalent in East African migrants to the UK; however severe cardiac ischaemia and liver damage can result.

Khat also known as qat or chat is the fresh leaves of Catha edulis. It is a shrub grown in East Africa and the Arabian Peninsula where khat chewing is common with open legal trade. Around 20 million people use khat in these countries and its use has spread to the immigrant communities across Europe and America. The 2001 census records 44000 Somalis in the UK and estimates of 95000 were made in 2003. On chewing, khat leaves release amphetamine-like constituents; cathinone and cathin and other alkaloids (cathulidins). Khat is currently legal in the UK though cathinone and cathin are controlled drugs (Misuse of Drugs Act 1971). Khat is illegal in the USA, Canada, Norway and Sweden.

\section{CASE HISTORY}

A 33-year-old East African man presented with acute onset chest pain. He smoked (15 pack years), but had no other coronary risk factors (cholesterol $4.8 \mathrm{mmol} / \mathrm{L}$ ). The patient had been chewing khat almost constantly for 2-3 days, but denied a regular habit; no reason for this change in behaviour was given. He took no medication and did not use illegal drugs.

His electrocardiogram (ECG) showed acute anterior myocardial infarction. After thrombolysis with rt-PA his pain resolved but the ECG showed no signs of reperfusion. Calcium channel antagonists were given intravenously for possible vasospasm. Troponin $\mathrm{T}$ at $12 \mathrm{~h}$ was $10.11 \mu \mathrm{g} / \mathrm{L}$ (normal range $<0.01$ ) indicating myocardial infarction. Transthoracic echocardiogram showed anteroseptal and apical akinesis with a moderately impaired left ventricle. The patient refused coronary angiography and received medical therapy of aspirin, an angiotensin converting enzyme inhibitor, a $\beta$-blocker and a statin.

The patient failed to attend for follow up having stated that he relied on his faith for his health. He returned 27 months later with shortness of breath at rest and

The Heart Hospital, London W1G 8PH, UK

Correspondence to: Dr Sagar Saha

E-mail: sagsaha@hotmail.com 
(a)
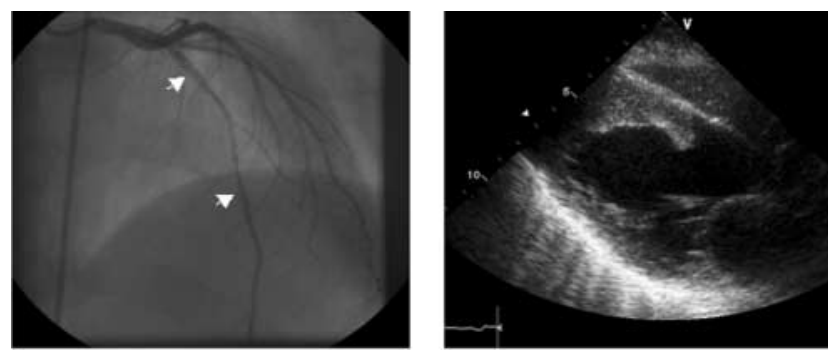

Figure 1 (a) Coronary angiogram showing $6 \mathrm{~cm}$ stenosis in the left anterior descending artery (arrows) filled with clot.

(b) Transthoracic echocardiogram (parasternal long axis view) showing a large apical left ventricle clot (arrows)

intermittent chest pain. In the interim, he had continued daily khat chewing. He was short of breath at rest, tachycardic, normotensive, and had a pansystolic murmur. He had signs of biventricular failure. Electrocardiogram showed his previous myocardial infarction without acute changes. Troponin $\mathrm{T}$ was $0.09 \mu \mathrm{g} / \mathrm{L}$. Echocardiography revealed severely impaired biventricular function (ejection fraction of $15 \%$, normal range $65-80 \%$ ).

Coronary angiography showed a $6 \mathrm{~cm}$ stenosis in the left anterior descending coronary artery and filling defects consistent with thrombus. The other coronary arteries were normal (Figure 1a). A dobutamine stress echocardiogram revealed no symptoms or electrocardiogram changes at peak stress and failed to show significant contractile reserve or viability suggesting an established infarct with no salvageable myocardium. In view of the negative stress echocardiogram, coronary intervention was not attempted and the patient was discharged on perindopril, carvedilol, frusemide, clopidogrel, simvastatin and spironolactone.

One month later, he returned with worsening chest pain and shortness of breath having stopped taking all his medications. He was hypotensive $(104 / 64 \mathrm{mmHg})$, his jugular venous pulse was elevated and his electrocardiogram was unchanged despite a Troponin $\mathrm{T}$ of $1.83 \mu \mathrm{g} / \mathrm{L}$. The patient had impaired liver function from admission, which continued to deteriorate with peak alanine transaminase of $626 \mathrm{IU} / \mathrm{L}$. Ultrasound showed an enlarged liver with reduced echogenicity consistent with acute hepatitis. A hepatitis screen was negative (B and $\mathrm{C}$, autoantibodies) as was an HIV test. The patient's left ventricle had deteriorated and echocardiography showed an extensive mural thrombus in the apex of his severely impaired left ventricle (Figure $1 b$ ). He was anticoagulated with warfarin and intravenous diuretics were required to treat fluid overload. An implantable cardioverter defibrillator was not attempted at this admission due to the risk of embolism from the left ventricle thrombus. Ultimately, the patient was discharged on maximal medical therapy after a 6-week stay to stabilize his severe biventricular failure.

\section{COMMENT}

Khat contains alkaloids cathulidins and cathinone which mediate its sympathomimetic effects. ${ }^{1}$ These release serotonin and dopamine in the central nervous system and noradrenaline from peripheral sympathetic neurones. ${ }^{1}$ Cathinone has a similar action to amphetamine and cocaine causing an elevation in blood pressure and heart rate proportional to blood levels which peak $1.5-3.5 \mathrm{~h}$ after chewing. ${ }^{2}$ Myocardial oxygen demand increases followed by catecholamine-mediated platelet aggregation and coronary vasospasm. ${ }^{3}$ We describe a case of severe ischaemic cardiomyopathy due to khat-related myocardial infarction.

A case-control study in the Yemen comparing 100 patients with acute myocardial infarction to 100 age- and sex-matched controls showed a 39-fold increased myocardial infarction risk in heavy khat chewers. In a multivariate analysis the relative risk of myocardial infarction associated with khat was 5.0 (confidence interval 1.9-13.1). ${ }^{4}$ Our patient's unusually sustained khat use for 2-3 days without sleep probably caused his extensive infarction.

No published guidelines exist for the optimal management of khat-induced coronary ischaemia; however, extrapolation from cocaine-associated myocardial infarction supports use of benzodiazepines, calcium channel blockers and nitrates with coronary intervention if these are unsuccessful.$^{5}$

To date, khat-associated human hepatotoxicty is unknown; but rabbit studies implicate long-term high dose khat use in chronic hepatic inflammation and porto-portal fibrosis with associated liver dysfunction. ${ }^{6}$ Our patient's hepatitis was is probably due to right heart failure and possible direct khat toxicity (it did not respond to withdrawal of statin or proton pump inhibitor).

Khat also causes gingivitis and tooth loss and may increase oesophageal cancer risk. ${ }^{7}$ Case reports implicate khat usage in memory impairment, depression and psychoses. ${ }^{7}$ The World Health Organization classifies khat as causing psychological, but not physical dependence. ${ }^{7}$

The practice of chewing khat leaves has been a part of the culture in areas of East Africa and the Arabian Peninsula since the 7th century. In these regions, khat is traded openly and is used socially across a range of age and class groups, particularly as alcohol is not permitted in Islam. It also has religious association and has been described as an aid to religious devotion and prayer. Migrants from these regions see khat chewing as integral to retaining their cultural and social identity. However, approval is not universal and varies with gender, region and generation; there is an awareness of the harmful effects on health and productivity. ${ }^{8}$ This differing of opinion was reflected in a survey by 
Nacro, a government drugs charity. This showed that in the UK, although $50 \%$ of Somali men regularly use khat and $35 \%$ of the 553 Somalis interviewed regarded it as important to cultural identity, $49 \%$ wanted to see it criminalized. ${ }^{9}$ The legal status of khat is currently under Home Office review. Although the harmful effects of chronic khat usage have been widely reported, the cultural effects of a ban on an immigrant minority need to be considered.

Our patient demonstrates its adverse effects on multiple organ systems including, not only the severe cardiovascular consequences of long-term khat use, but also its possible hepatotoxicity. Increased medical awareness of khat's availability and use by urban migrant populations in the UK is needed to allow prompt treatment of coronary ischaemia, particularly in young male khat chewers with few other risk factors for coronary disease.

Acknowledgments We would like to acknowledge Dr Malcolm Walker's contribution to this patient's care and preparation of this report.

Competing interests None.

\section{REFERENCES}

1 Kalix P. A comparison of the catecholamine releasing effect of the khat alkaloids (-)-cathinone and (+)-norpseudoephidrine. Drug Alcohol Depend 1983;11:395-401

2 Halket JM, Karasu Z, Murray-Lyon IM. Plasma cathinone levels following chewing khat leaves. J Ethnoparmacol 1995;49:111-13

3 Al-Motarreb AL, Broadly KJ. Coronary and aortic vasoconstriction by cathinone, the active constituent of khat. Auton Autacoid Pharmacol 2003; 23:319-26

4 Al-Motarreb AL, Briancon S, Al-Jaber N, et al. Khat chewing is a risk factor for acute myocardial infarction: a case control study. $\mathrm{Br} J \mathrm{Clin}$ Pharmacol 2005;59:574-81

5 Braunwald E, Antman E, Beasley J, et al. ACC/AHA 2002 guideline update for the management of patients with unstable angina and non-STsegment elevation infarction. J Am Coll Cardiol 2002;40:1366-74

6 Al-Habori M, Al-Aghbari A, Al-Mamary M, et al. Toxicological evaluation of Catha edulis leaves: a long term feeding experiment in animals. J Ethnopharmacol 2002;83:209-17

7 Houghton P. Khat - a growing concern in the UK. Pharmaceutical J $2004 ; 272: 163-5$

8 8. Stevenson M, Fitzgerald J, Banwell C. Chewing as a social act: cultural displacement and khat consumption in the East African communities of Melbourne. Drug Alcohol Rev 1996;15:73-82

9 Patel SL, Murray R. Khat chewing amongst Somalis in four English cities. [http://www.homeoffice.gov.uk/rds/pdfs05/r266.pdf]

\section{Oesophageal lichen planus: a missed diagnosis}

\author{
Abdul R Mohammed ${ }^{1} \quad$ Paul Sherwood ${ }^{2}$
}

J R Soc Med 2006:99:318-320

Lichen planus is a chronic inflammatory mucocutaneous disease of unknown aetiology with no racial predisposition, although there is a considerable variation in its incidence. Involvement of oesophagus is considered to be extremely rare; the diagnosis is often not made until complications occur. An epidemiological study of 584 patients with oral lichen planus showed oesophageal involvement in only six patients, clearly showing a rare prevalence. The incidence of this disease in dermatology clinics is $1.4 \%$, compared to $3 \%$ in oral medicine clinics. We describe a 69 -year-old woman who presented with a 2-year history of odynophagia due to oesophageal lichen planus. This case is very unusual in that she had skin involvement 28 years before and had no recurrence.

\section{CASE HISTORY}

A 69-year-old retired midwife was referred to the gastroenterology clinic by her general practitioner with a 2-year history of progressive odynophagia. It occurred especially if she ate bulky foods such as bread and potatoes. She was only able to eat extremely slowly and occasionally had choking episodes. Antacids were ineffective, and lansoprazole caused intolerable colic and diarrhoea. Eighteen months previously she had a normal barium swallow and fibreoptic laryngoscopy. She had suffered extensive lichen planus of the skin in 1977 for which she received treatment for 3 months, and had complete remission within a few months. She had no further skin involvement. Subsequently, she had oral lichen planus diagnosed by her dentist 4 years previously, which subsided without treatment. She was not on any regular medications when the referral was made to us. Interestingly, her sister had been diagnosed with lichen planus of the skin a few weeks before. An outpatient gastroscopy was organized.

During gastroscopy a lacy network of white lines was visible in the oesophagus representing Wickham's striae of lichen planus (Figure 1). The upper third of oesophagus felt tight and 'gripped' the scope. The lower oesophagus looked

${ }^{1}$ Senior SHO in Internal Medicine; ${ }^{2}$ Consultant Gastroenterologist, Northampton General Hospital, Northampton NN1 5BD, UK

Correspondence to: Dr Abdul R Mohammed, Specialist Registrar, Department of Gastroenterology, Addenbrooke's Hospital, Cambridge CB2 2QQ, UK

E-mail: drmohdrafi@doctors.net.uk 


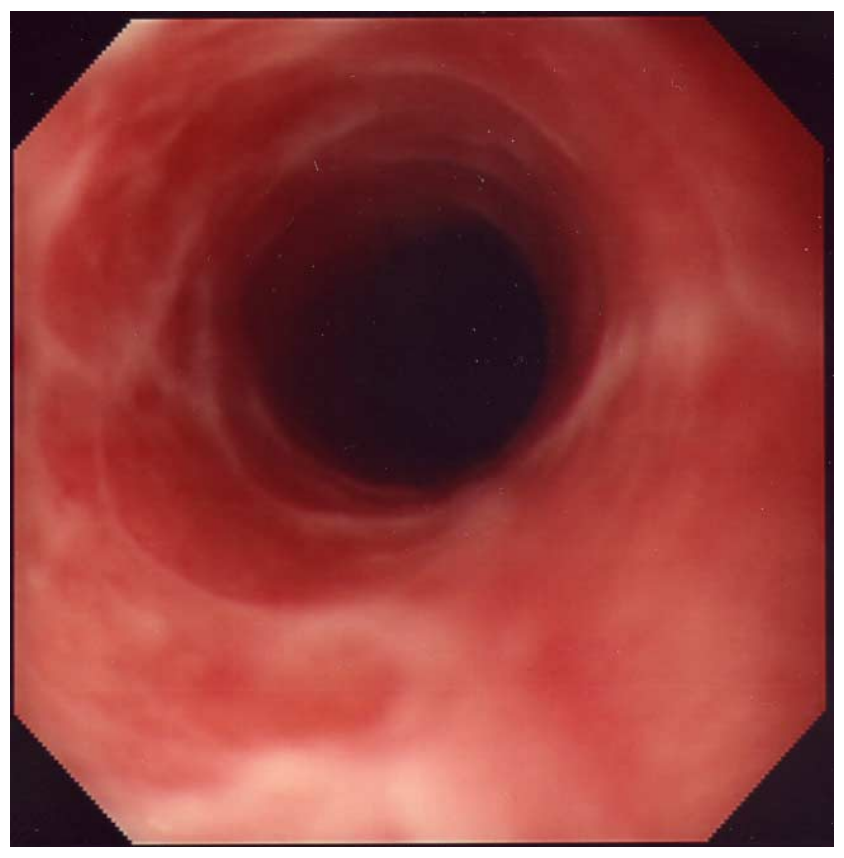

Figure 1 Endoscopic view of oesophageal lichen planus

normal except that she had a small $(<5 \mathrm{~cm})$ sliding hiatus hernia. Oesophageal candidiasis was considered as a possibility, but the appearances were not typical of this diagnosis and there were no risk factors for it. Multiple upper oesophageal biopsies were taken, but, to our dismay at this stage, the histopathologist reported that the features were consistent with reflux oesophagitis and that there were no features to suggest lichen planus or candida infection. As per the histopathology report, the patient was put on esomeprazole $40 \mathrm{mg}$ once daily. She was followed up 4 weeks later and reported that her symptoms were worse than before. A repeat gastroscopy performed 9 weeks later whilst she was taking esomeprazole revealed the same macroscopic findings as before. Multiple biopsies were again taken from upper oesophagus and the histology showed patchy basal degeneration of squamous epithelium. There was also evidence of dense infiltration of lymphocytes with an occasional Civatte body within squamous epithelium, features consistent with oesophageal lichen planus. Subsequently she has been put on oral prednisolone 40mg per day for 2 weeks, reducing by $5 \mathrm{mg}$ per fortnight. She is currently taking $5 \mathrm{mg}$ per day and reports $85 \%$ improvement in odynophagia.

\section{DISCUSSION}

Lichen planus is a chronic inflammatory mucosal disease of unknown aetiology affecting skin, mouth or genitals. Its association with HLA-BW 16, B8, and DR1 suggests the possibility of genetic predisposition. ${ }^{1}$ It is characterized by shiny, violaceous, flat-topped polygonal papules which retain the skin lines, and which vary in size from pinpoint to a centimetre or more across. ${ }^{2}$ White lines, known as Wickham's striae may traverse the surface of the papules. Linear lesions often appear along scratch marks or in scars (Koebner phenomenon). Skin lesions may be disfiguring, and involvement of the oral mucosa or genital mucosa in severe cases can be debilitating. While most cases of lichen planus are idiopathic, some may be caused by the ingestion of certain medications (e.g., gold, antimalarial agents, penicillamine, thiazides, beta blockers, nonsteroidal anti-inflammatory drugs, quinidine and angiotensin-converting enzyme inhibitors) or linked to hepatitis $\mathrm{C}$ virus infection. ${ }^{3}$ Diabetes mellitus is a possible associated disease of oral lichen planus ${ }^{4}$ and candidiasis may also coexist in some patients.

The well-known histopathological features of lichen planus are compact orthokeratosis, wedge shaped hypergranulosis, vacuolar alteration of the basal layer, necrotic keratinocytes (so-called Civatte bodies) and a dense bandlike infiltrate composed mostly of lymphocytes. ${ }^{5}$ Not all of these features must be present to establish the diagnosis of lichen planus.

Oesophageal lichen planus is said to be often unrecognized and underestimated. ${ }^{6}$ It should always be evaluated for in patients with mucocutaneous involvement. The overwhelming clinical presentation is dysphagia; but reflux-type symptoms, or retrosternal pain, can also be associated. Delay in diagnosis may lead to serial dilations of oesophageal strictures that, without simultaneous medical treatment, may lead to koebenerization of lichen planus and worsening of the stricture. Patients can lose significant weight and become dehydrated secondary to stenosis. Squamous cell cancer developing on mouth lesions is uncommon, but is a potential danger, especially with ulcerated lesions. ${ }^{2}$ No case has yet been reported in oesophageal lichen planus. After the initial diagnosis of oesophageal lichen planus is made, systemic steroids are often needed to quell the inflammation. A variety of steroid sparing agents have been used successfully, including azathioprine, cyclosporine and etretinate. ${ }^{7}$ Most patients with symptomatic disease initially respond to immunosuppressant therapy, but recurrent stenosis, in spite of treatment, is common and repeated endoscopic dilatations are necessary in the long run. ${ }^{8}$

Recognition of oesophageal lichen planus is critical for several reasons. Misinterpretation of the histological features as secondary to reflux disease or as simply a nonspecific reaction can lead to delay in the diagnosis and the continuation or worsening of the symptoms. ${ }^{7}$ It outlines the importance of a proper history and full examination to look for any potential extra oral manifestations of lichen planus. Although no case of carcinoma developing in the setting of oesophageal lichen planus has been reported, some authors think such patients should be followed endoscopically, even prospectively evaluated, for this possibility. ${ }^{9}$ 


\section{CONCLUSION}

Oesophageal lichen planus is a rare cause of oesophageal symptoms in patients with no active skin or oral lichen planus. A history of lichen planus should be sought in patients presenting with oesophageal symptoms.

\section{REFERENCES}

1 Valsecchi R, Bontempelli M, Rossi A, et al. HLA-DR and DQ antigens in lichen planus. Acta Derm Venerol (Stockh) 1988;68:77-80

2 Breathnach SM, Black MM. Lichen planus and lichenoid reactions. In: Burns T, Breathnach SM, Cox N, Griffiths C, eds. Rook's Textbook of Dermatology, 7th edn. Oxford: Blackwell Science, 2004:42.6

3 Katta R. Lichen planus. Am Fam Physician. 2000;61:3319-24
4 Smith MJA. Oral lichen planus and diabetes mellitus. J Oral Med 1977;32: 110-12

5 Acherman AB. Histologic Diagnosis of Inflammatory Skin Diseases. Philadelphia: Lea \& Febiger, 1978

6 Eisen D. The evaluation of cutaneous, genital, scalp, nail, oesophageal, and ocular involvement in patients with oral lichen planus. Oral Surg Oral Med Oral Pathol 1999;88:431-6

7 Menges M, Hohloch K, Pueschel W, Stallmach A. Lichen planus with oesophageal involvement: a case report and review of the literature. Digestion 2002;65:184-9

8 Abraham SC, Ravich WJ, Anhalt GJ, Yardley JH, Tsung-Teh W. Oesophageal Lichen planus: case report and review of the literature. Am J Surg Pathol 2000;24:1678-82

9 Dickens CM, Heseltine D, Walton S, Bennett JR. The oesophagus in lichen planus: an endoscopic study. BMJ 1990;300:84 